\title{
Batched Evaluation of Full-Sharing Multithreaded Tabling
}

\author{
Miguel Areias and Ricardo Rocha \\ CRACS \& INESC TEC and Faculty of Sciences, University of Porto \\ Rua do Campo Alegre, 1021, 4169-007 Porto, Portugal \\ \{miguel-areias, ricroc\}@dcc.fc.up.pt
}

\begin{abstract}
Tabling is a technique that overcomes some limitations of traditional Prolog systems in dealing with redundant sub-computations and recursion. When tabling is combined with multithreading, we have the best of both worlds, since we can exploit the combination of higher declarative semantics with higher procedural control. To support this combination, the Yap Prolog system has, at engine level, multiple designs that vary from a No-Sharing design, where each thread allocates fully private tables, to a Full-Sharing (FS) design, where threads share the complete table space. In this work, we propose an extension to the table space data structures, which we named Private Answer Chaining $(P A C)$, as way to support batched scheduling evaluation with the FS design. Batched scheduling is one of the most successful tabling scheduling strategies, known to be useful when a tabled logic program requires an eager propagation of answers and/or do not requires the complete set of answers to be found. Experimental results show that PAC is a good first approach, since with it the FS design remains quite competitive.
\end{abstract}

Keywords: Logic Programming, Multithreading, Tabling, Scheduling.

\section{Introduction}

Tabling [5] is a technique that overcomes some limitations of traditional Pro$\log$ systems in dealing with redundant sub-computations and recursion. Tabling consists in storing intermediate answers for subgoals in a proper data structure, called the table space, so that they can be reused when a repeated subgoal appears during the resolution process. Tabling has become a popular and successful technique thanks to the ground-breaking work in the XSB Prolog system and in particular in the SLG-WAM engine [10], the most successful engine of XSB. Implementations of tabling are now widely available in systems like Yap Prolog, B-Prolog, ALS-Prolog, Mercury, Ciao Prolog and more recently Picat.

Multithreading in Prolog is the ability to concurrently perform computations, in which each computation runs independently but shares the program clauses. When multithreading is combined with tabling, we have the best of both worlds, since we can exploit the combination of higher procedural control with higher declarative semantics. To the best of our knowledge, XSB [8] 
and Yap [2] are the only Prolog systems that support the combination of multithreading with tabling. In this work, we will focus on Yap's implementation, which follows a SWI-Prolog compatible multithreading library [11]. For tabled evaluation, a thread views its tables as private but, at the engine level, Yap has three designs [2], which vary from a No-Sharing (NS) design, where each thread allocates private tables for each new tabled subgoal call, to a Full-Sharing (FS) design, where threads share the complete table space.

The decision about the evaluation flow is determined by the scheduling strategy. Different strategies may have a significant impact on performance, and may lead to a different ordering of solutions to the query goal. Arguably, the two most successful tabling scheduling strategies are local scheduling and batched scheduling [6]. Local scheduling tries to complete subgoals as soon as possible. When new answers are found, they are added to the table space and the evaluation fails. Local scheduling has the advantage of minimizing the size of clusters of dependent subgoals, however it delays propagation of answers and requires the complete evaluation of the search space.

Batched scheduling favors forward execution first, backtracking next, and consuming answers or completion last. It thus tries to delay the need to move around the search tree by batching the return of answers to repeated subgoals. When new answers are found for a particular tabled subgoal, they are added to the table space and the evaluation continues. Batched scheduling can be an useful strategy in tabled logic programs that require an eager propagation of answers and/or do not require the complete set of answers to be found.

With the FS design, all tables are shared. Thus, since several threads can be inserting answers in the same table, when an answer already exists, it is not possible to determine if the answer is new or repeated for a particular thread without further support. For local scheduling, this is not a problem since, for repeated and new answers, local scheduling always fails. The problem is with batched scheduling that requires that only the repeated answers should fail. Threads have then to detect, during batched evaluation, whether an answer is new and must be propagated or whether an answer is repeated and the evaluation should fail.

In this work, we propose an extension to the table space data structures, which we named Private Answer Chaining (PAC), as a way to keep track, per thread and subgoal call, of the answers that were already found and propagated. We discuss in detail our proposal for extending the FS design with batched scheduling and we present a performance analysis comparison between local and batched scheduling. Experimental results show that, despite the extra PAC data structures required to support batched scheduling with the FS design, the execution time of the combination is still quite competitive.

The remainder of the paper is organized as follows. First, we briefly introduce some background and related work. Then, we describe our PAC approach and we discuss the most important implementation details. Finally, we present experimental results and we end by outlining some conclusions. 


\section{Background}

The basic idea behind tabling is straightforward: programs are evaluated by storing answers for tabled subgoals in an appropriate data space, called the table space. Repeated calls ${ }^{1}$ to tabled subgoals are not re-evaluated against the program clauses, instead they are resolved by consuming the answers already stored in their table entries. During this process, as further new answers are found, they are stored in their tables and later returned to all repeated calls.

Figure 1 shows Yap's table space organization. At the entry point we have the table entry data structure. This structure is allocated when a tabled predicate is being compiled, so that a pointer to the table entry can be included in its compiled code. This guarantees that further calls to the predicate will access the table space starting from the same point. Below the table entry, we have the subgoal trie structure. Each different tabled subgoal call to the predicate at hand corresponds to a unique path through the subgoal trie structure, always starting from the table entry, passing by several subgoal trie data units, the subgoal trie nodes, and reaching a leaf data structure, the subgoal frame. The subgoal frame stores additional information about the subgoal

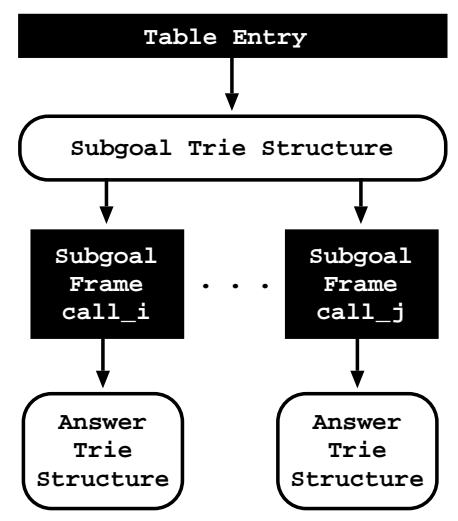

Fig. 1. Table space organization and acts like an entry point to the answer trie structure. Each unique path through the answer trie data units, the answer trie nodes, corresponds to a different answer to the entry subgoal.

\subsection{Yap's Multithreaded Tabling Support}

In Yap, a thread views its tables as private but, at the engine level, it implements three designs for concurrent tabling support that vary from a No-Sharing (NS) design, where each thread allocates fully private tables, to a Full-Sharing (FS) design, where threads share the complete table space. Figure 2 shows Yap's multithreaded table space organization for the NS and FS designs, where an interface layer abstracts the design being used at the engine level. The figure illustrates the main differences between the two designs for a situation where several threads are evaluating the same tabled subgoal call call_ $i$.

When using the NS design, one can observe that the table entry data structure still stores the common information for the predicate (such as the arity or the scheduling strategy), and then each thread $t$ has its own cell $T_{t}$ inside a bucket array which points to the private data structures.

\footnotetext{
${ }^{1}$ We are considering variant-based tabling [9]. Two tabled subgoals A and B are variants if they can be made identical by variable renaming. For example, $p(X, 1, Y)$ and $p(Y, 1, Z)$ are variants because both can be transformed into $p\left(V A R_{0}, 1, V A R_{1}\right)$.
} 


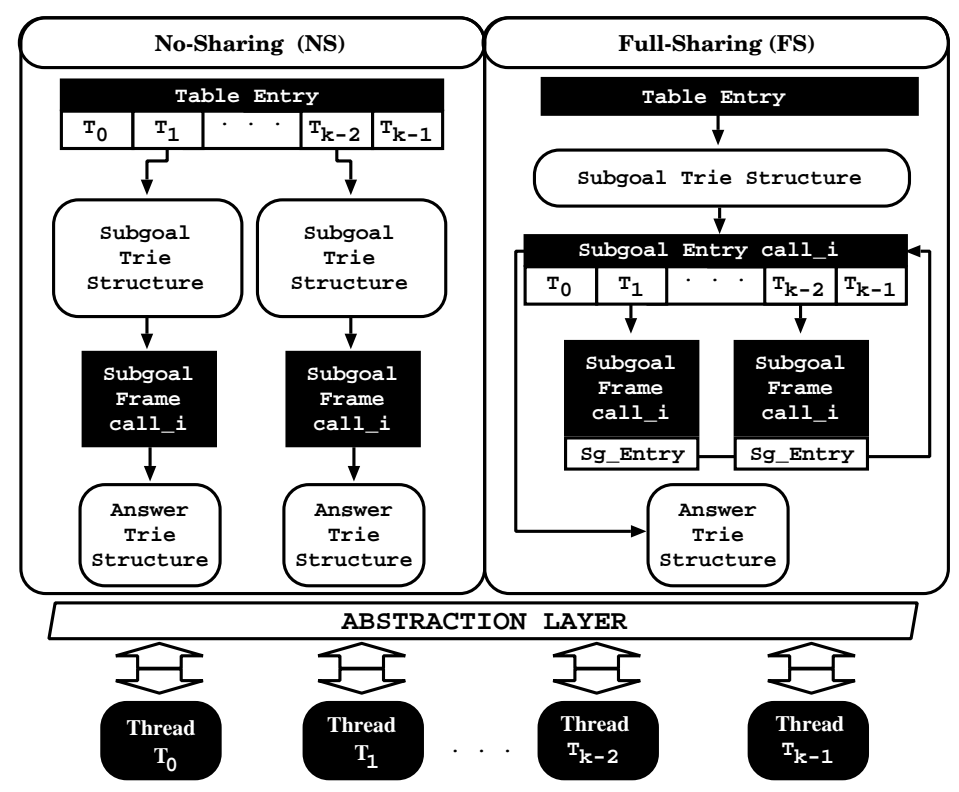

Fig. 2. Yap's multithreaded table space organization for the NS and FS designs

When using the FS design, the subgoal and answer trie structures and part of the subgoal frame (the subgoal entry data structure in Fig. 2) are shared among all threads. The previous subgoal frame data structure was split in two: the subgoal entry stores common information for the subgoal call (such as the pointer to the shared answer trie structure); the remaining information is kept private to each thread in the subgoal frame data structure. To support concurrency within the subgoal/answer tries, the FS design supports lock-based and lockfree approaches. A comparison between both approaches can be found in [3].

\subsection{Scheduling Strategies}

Local scheduling evaluates a tabled logic program in a breath-first manner. It favors backtracking first with completion instead of forward execution, leaving the consumption of answers for last. Local scheduling only allows a Cluster of Dependent Subgoals $(C D S)$ to return answers after a fix-point has been reached [6]. In other words, local scheduling tries to keep a CDS as minimal as possible, thus creating less complex dependencies between subgoals, which causes a sooner completion of subgoals.

On the other hand, batched scheduling evaluates a tabled logic program in a depth-first manner. It favors forward execution first instead of backtracking, leaving the consumption of answers and completion for last. It thus tries to delay the need to move around the search tree by batching the return of answers. When new answers are found for a particular tabled subgoal, they are added to the 
table space and the execution continues. For some situations, this results in creating dependencies to older subgoals, therefore enlarging the current CDS and delaying the fix-point that guarantees that all dependent subgoals in a CDS are completely evaluated [10]. Batched scheduling can be an useful strategy in tabled logic programs that require an eager propagation of answers and/or do not require the complete set of answers to be found.

\section{Extending Full-Sharing with Batched Scheduling}

In this section, we describe our proposal to support the combination of batched scheduling with the FS design. In the original FS design, answer propagation and answer representation are both stored in the answer trie data structure, thus threads are unable to distinguish whether they have or not have propagated an answer already stored in the table space. To solve that, we propose an extension to the table space data structures, which we named Private Answer Chaining (PAC), as a way to keep track, per thread and subgoal call, of the answers that were already found and propagated to the thread's repeated calls. Figure 3 illustrates PAC's key idea. In a nutshell, PAC splits answer propaga-

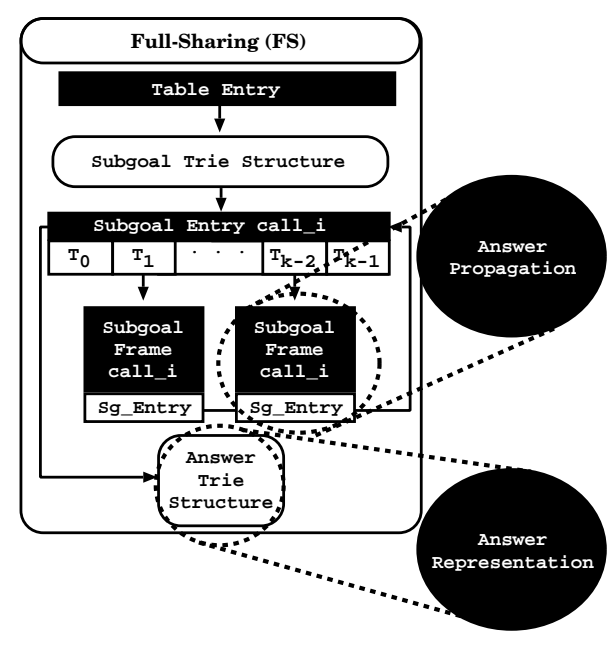

Fig. 3. PAC overview tion from answer representation, and allows the first to be privately stored in the subgoal frame data structure of each thread, and the second to be kept publicly shared among threads in the answer trie data structure.

\subsection{Our Approach}

The PAC procedure works at the subgoal frame level. The key idea is to extend subgoal frames with an auxiliary private chaining of answers for each subgoal call, in order to keep track of the answers already found for the call. Later, if a thread completes a subgoal's evaluation, i.e, if the subgoal's table is marked as complete, its PAC is made public, so that from that point on all threads can use that chain in complete (only reading) mode. Figure 4 illustrates the new data structures involved in the implementation of our PAC's proposal for a situation where different threads are evaluating the same tabled subgoal call call_ $i$.

Figure 4 (a) shows then a situation where two threads, $T_{1}$ and $T_{k-2}$, are sharing the same subgoal entry for a call call $i$ still under evaluation, i.e., still not yet completed. The current state of the evaluation shows an answer trie with 


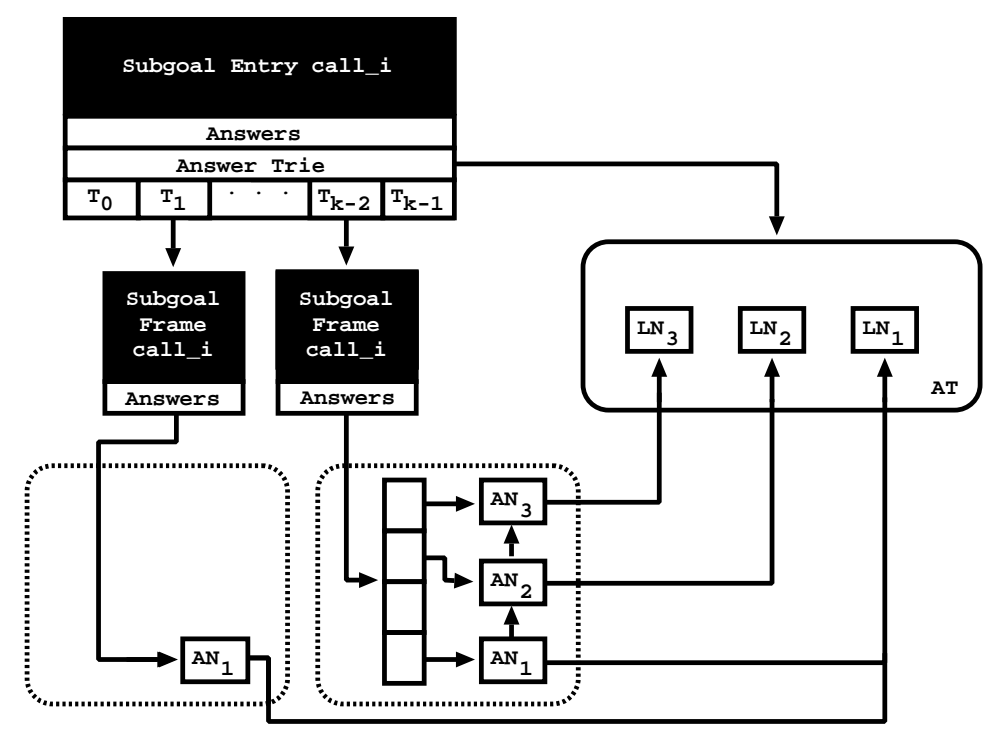

(a)

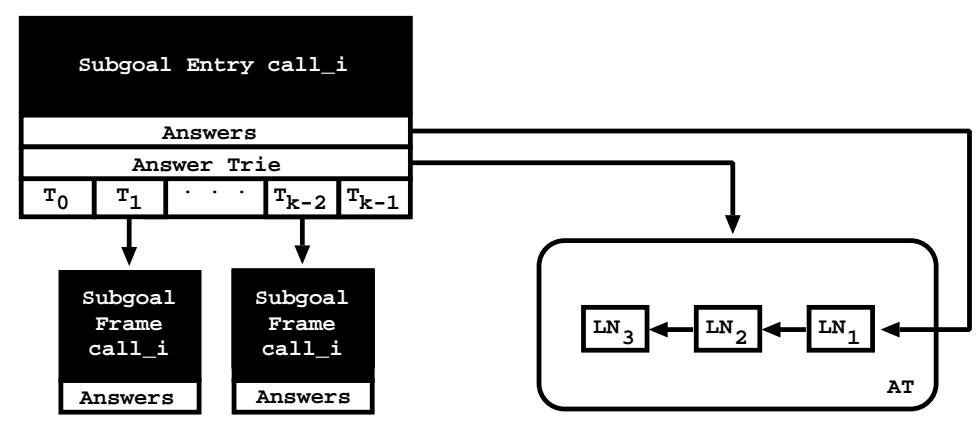

(b)

Fig. 4. PAC's data structures for (a) private and (b) public chaining

3 answers found for call $i$. For the sake of simplicity, we are omitting the internal answer trie nodes and we are only showing the leaf nodes $L N_{1}, L N_{2}$ and $L N_{3}$ of each answer.

With PAC support, the leaf nodes are not chained in the answer trie data structure, as usual. Now, the chaining process is done privately, and for that, we use the subgoal frame structure of each thread. On the subgoal frame structure we added a new field, called Answers, to store the answers found within the execution of the thread. In order to minimize PAC's impact, each answer node in the private chaining has only two fields: (i) an entry pointer, which points to the corresponding leaf node in the answer trie data structure; and (ii) a next pointer to chain the nodes in the private chaining. To maintain good performance, when 
the number of answer nodes exceeds a certain threshold, we use a hash trie mechanism design similar to the one presented in [4], but without concurrency support, since this mechanism is private to each thread.

PAC's data structures in Fig. 4(a) represent then two different situations. Thread $T_{1}$ has only found one answer and it is using a direct answer chaining to access the leaf node $L N_{1}$. Thread $T_{k-2}$ was already found three answers for call_ $i$ and it is using the hash trie mechanism within its private chaining. In the hash trie mechanism, the answer nodes are still chained between themselves, thus that repeated calls belonging to thread $T_{k-2}$ can consume the answers as in the original mechanism.

Figure 4(b) shows the state of the subgoal call after completion. When a thread $T$ completes a subgoal call, it frees its private consumer structures, but before doing that, it checks whether another thread as already marked the subgoal as completed. If no other thread has done that, then thread $T$ not only follows its private chaining mechanism, as it would for freeing its private nodes, but also follows the pointers to the answer trie leaf nodes in order to create a chain inside the answer trie. Since this procedure is done inside a critical region, no more than one thread can be doing this chaining process. Thus, in Fig. 4(b), we are showing the situation where the subgoal call call_ $i$ is completed and both threads $T_{1}$ and $T_{k-2}$ have already chained the leaf nodes inside the answer trie and removed their private chaining structures.

\subsection{Implementations Details}

The major difference between local and batched scheduling, at the engine level, is in the tabled new answer operation, where we decide what to do when a new answer is found during the evaluation. This operation checks whether a newly found answer is already in the corresponding answer trie structure and, if not, inserts it. For local scheduling, it then fails and, for batched scheduling, it proceeds with forward execution. Algorithm 1 shows how we have extended this operation to support the FS design with batched scheduling.

The algorithm receives two arguments: the newly found answer during the evaluation $(A N S)$ and the subgoal frame which corresponds to the call at hand $(S F)$. The algorithm begins by checking/inserting the given $A N S$ into the answer trie structure, which will return the leaf node for the path representing $A N S$ (line 1). Then, it checks/inserts the given leaf node into the private chaining for the current thread, which will return the corresponding answer chain node (line 2). Next in line 3, it tests whether the answer chain node already existed in the chain, i.e., if it was inserted or not by the current check/insert operation in order to return failure (line 4), or it proceeds with marking the answer $A N S$ has found (line 6). At the end (lines 7 to 10), it returns failure, if local scheduling is active (line 8), otherwise, batched scheduling is active, and it proceeds by propagating the answer $A N S$ to the current execution environment (line 10). 


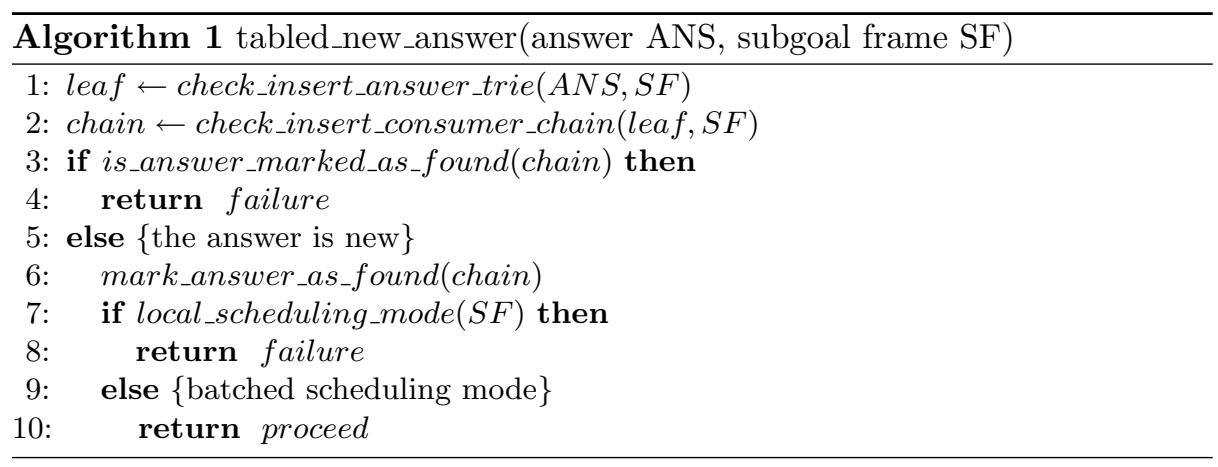

\section{Experimental Results}

We now present experimental results about the usage of PAC in the FS design with batched scheduling. The environment for our experiments was a machine with 32-Core AMD Opteron (TM) Processor 6274 (2 sockets with 16 cores each) with 32GB of main memory, running the Linux kernel 3.16.7-200.fc20.x86_64 with Yap Prolog 6.3.

\subsection{Benchmark Programs}

For the experiments, we used the TabMalloc memory allocator [1] and five sets of benchmarks that create worst case scenarios, where we are able to show the lowest bounds of performance that each design might achieve when applied/used in other real world applications/programs. The Large Joins and WordNet sets were obtained from the OpenRuleBench project [7]; the Model Checking set includes three different specifications and transition relation graphs usually used in model checking applications; the Path Left and Path Right sets implement two recursive definitions of the well-known path/2 predicate, that computes the transitive closure in a graph, using several different configurations of edge/2 facts (Fig. 5 shows an example for each configuration). We experimented the BTree configuration with depth 17, the Pyramid and Cycle configurations with depth 2000 and the Grid configuration with depth 35.

In order to have a deeper insight on the behavior of each benchmark, and therefore clarify some of the results that are presented next, we first characterize the benchmarks. The columns in Table 1 have the following meaning:

- calls: is the number of different calls to tabled subgoals. It corresponds to the number of paths in the subgoal tries.

- trie nodes: is the total number of trie nodes allocated in the corresponding subgoal/answer trie structures.

- trie depth: is the minimum/average/maximum number of trie nodes required to represent a path in the corresponding subgoal/answer trie structures. Trie structures with smaller average depth values are more amenable to higher contention. 


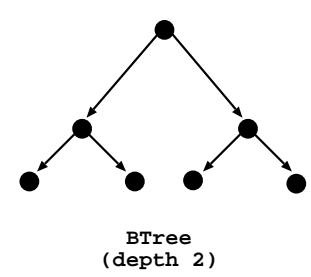

(depth 2)

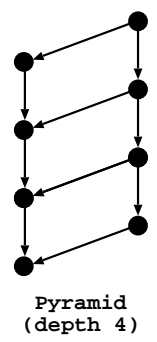

(depth 4)

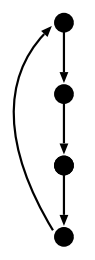

Cycle
(depth 4)

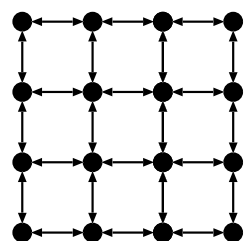

Grid

Fig. 5. Edge configurations for the path benchmarks

- unique: is the number of different tabled answers found. It corresponds to the number of paths in the answer tries.

- repeated: is the number of redundant tabled answers found.

By observing Table 1, the Mondial benchmark, from the Large Joins set, and the three Model Checking benchmarks seem to be the benchmarks least amenable to contention since they are the ones that find less unique answers and that have the deepest trie structures. In this regard, the Path Left and Path Right sets correspond to the opposite case. They find a huge number of answers and have very shallow trie structures. On the other hand, the WordNet and Path Right sets have the benchmarks with the largest number of different subgoal calls, which can reduce the probability of contention because answers can be found for different subgoal calls and therefore be inserted with minimum overlap. On the opposite side are the Join2 benchmark, from the Large Joins set, and the Path Left benchmarks, which have only a single tabled subgoal call.

\subsection{Performance Analysis}

We present now the performance analysis about the usage of PAC in the FS design with batched scheduling. To support concurrency within the subgoal/answer tries, the FS design is using the lock-free hash trie design presented in [3]. Since without PAC the FS design would not be able to be used with batched scheduling, to put PAC's results in perspective, we will be showing also the results for local scheduling and for the NS design.

Table 2 shows the overhead ratios for the five sets of benchmarks, when comparing against the NS design with 1 thread (running with local scheduling and without TabMalloc), for the NS and FS designs with 1, 8, 16, 24 and 32 threads, using local scheduling (column Local) and batched scheduling (column Batched) strategies with TabMalloc. In order to give a fair weight to each benchmark, the overhead ratio is calculated as follows. We begin by running 10 times each benchmark $B$ for each design $D$ with $T$ threads. Then, we calculate the average of those ten runs and use that value $\left(D_{B T}\right)$ to put it in perspective against the base time, which is the average of 10 runs of the NS design with 1 thread $\left(N S_{B 1}\right)$. For that, we use the following formula for the overhead $O_{D B T}=D_{B T} / N S_{B 1}$. 
Table 1. Characteristics of the benchmark programs

\begin{tabular}{|c|c|c|c|c|c|c|c|}
\hline \multirow{2}{*}{ Bench } & \multicolumn{3}{|c|}{ Tabled Subgoals } & \multicolumn{4}{|c|}{ Tabled Answers } \\
\hline & calls & \multicolumn{2}{|c|}{ trie nodes trie depth } & \multirow[t]{2}{*}{ unique } & \multicolumn{3}{|c|}{ repeated trie nodes trie depth } \\
\hline \multicolumn{7}{|c|}{ Large Joins } & \\
\hline Join2 & 1 & 6 & $5 / 5 / 5$ & $2,476,099$ & 0 & $2,613,660$ & $5 / 5 / 5$ \\
\hline Mondial & 35 & 42 & $3 / 4 / 4$ & 2,664 & $2,452,890$ & 14,334 & $6 / 7 / 7$ \\
\hline \multicolumn{8}{|l|}{ WordNet } \\
\hline Clusters & 117,659 & 235,319 & $2 / 2 / 2$ & 166,877 & 161,853 & 284,536 & $1 / 1 / 1$ \\
\hline Нуро & 117,657 & 117,659 & $2 / 2 / 2$ & 698,472 & 20,341 & 816,129 & $1 / 1 / 1$ \\
\hline Holo & 117,657 & 235,315 & $2 / 2 / 2$ & 74,838 & 54 & 192,495 & $1 / 1 / 1$ \\
\hline Hyper & 117,657 & 235,315 & $2 / 2 / 2$ & 698,472 & 8,658 & 816,129 & $1 / 1 / 1$ \\
\hline Tropo & 117,657 & 235,315 & $2 / 2 / 2$ & 472 & 0 & 118,129 & $1 / 1 / 1$ \\
\hline Mero & 117,657 & 117,659 & $2 / 2 / 2$ & 74,838 & 13 & 192,495 & $1 / 1 / 1$ \\
\hline \multicolumn{8}{|c|}{ Model Checking } \\
\hline IProto & 1 & 6 & $5 / 5 / 5$ & 134,361 & 385,423 & $1,554,896$ & $4 / 51 / 67$ \\
\hline Leader & 1 & 5 & $4 / 4 / 4$ & 1,728 & 574,786 & 41,788 & $15 / 80 / 97$ \\
\hline Sieve & 1 & 7 & $6 / 6 / 6$ & 380 & $1,386,181$ & 8,624 & $21 / 53 / 58$ \\
\hline \multicolumn{8}{|l|}{ Path Left } \\
\hline BTree & 1 & 3 & $2 / 2 / 2$ & $1,966,082$ & 0 & $2,031,618$ & $2 / 2 / 2$ \\
\hline Pyramid & 1 & 3 & $2 / 2 / 2$ & $3,374,250$ & $1,124,250$ & $3,377,250$ & $2 / 2 / 2$ \\
\hline Cycle & 1 & 3 & $2 / 2 / 2$ & $4,000,000$ & 2,000 & $4,002,001$ & $2 / 2 / 2$ \\
\hline Grid & 1 & 3 & $2 / 2 / 2$ & $1,500,625$ & $4,335,135$ & $1,501,851$ & $2 / 2 / 2$ \\
\hline \multicolumn{8}{|c|}{ Path Right } \\
\hline BTree & 131,071 & 262,143 & $2 / 2 / 2$ & $3,801,094$ & 0 & $3,997,700$ & $1 / 2 / 2$ \\
\hline Pyramid & 3,000 & 6,001 & $2 / 2 / 2$ & $6,745,501$ & $2,247,001$ & $6,751,500$ & $1 / 2 / 2$ \\
\hline Cycle & 2,001 & 4,003 & $2 / 2 / 2$ & $8,000,000$ & 4,000 & $8,004,001$ & $1 / 2 / 2$ \\
\hline Grid & 1,226 & 2,453 & $2 / 2 / 2$ & $3,001,250$ & $8,670,270$ & $3,003,701$ & $1 / 2 / 2$ \\
\hline
\end{tabular}

After calculating all the overheads $O_{D B T}$ for a certain design $D$ and number of threads $T$ corresponding to the several benchmarks $B$, we calculate the respective minimum, average, maximum and standard deviation overhead ratios (rows Min, Avg, Max and StD in Table 2).

By observing Table 2, we can see that batched scheduling always achieves the best minimum overhead ratio in the FS design but, for the average and maximum overhead ratios, the best strategy is always local scheduling. For the average and maximum overhead ratios, the difference between local and batched scheduling in the FS design is slightly higher than in the NS design, which can be read as an indication of the overhead that PAC introduces into the FS design. Recall that whenever an answer is found during the evaluation, PAC requires that threads traverse their private consumer data structures to check if the answer was already found (and propagated).

As we increase the number of threads, for the NS design, both scheduling strategies show very close minimum, average and maximum overhead ratios. For the FS design, the differences are slightly higher. However, for the average overhead ratio, the results between both strategies are quite close, with batched scheduling being around 10\% slower than local scheduling for the FS design. In summary, our experimental results show that, on average, the PAC strategy 
Table 2. Overhead ratios, when compared with the NS design with 1 thread (running with local scheduling and without TabMalloc) for the NS and FS designs (with TabMalloc) when running 1, 8, 16, 24 and 32 threads with local and batched scheduling (best ratios by row and design for the Minimum, Average and Maximum are in bold)

\begin{tabular}{|c|c|c|c|c|c|}
\hline \multirow{2}{*}{\multicolumn{2}{|c|}{ Threads }} & \multicolumn{2}{|c|}{ NS } & \multicolumn{2}{|c|}{ FS } \\
\hline & & Local & Batched & Local & Batched \\
\hline \multirow{4}{*}{1} & Min & 0.53 & 0.55 & 1.01 & 0.95 \\
\hline & Avg & 0.78 & 0.82 & 1.30 & 1.46 \\
\hline & Max & 1.06 & 1.05 & 1.76 & 2.33 \\
\hline & StD & 0.15 & 0.14 & 0.22 & 0.44 \\
\hline \multirow{4}{*}{8} & Min & 0.66 & 0.63 & 1.16 & 0.99 \\
\hline & Avg & 0.85 & 0.88 & 1.88 & 1.95 \\
\hline & Max & 1.12 & 1.14 & 2.82 & 3.49 \\
\hline & StD & 0.13 & 0.14 & 0.60 & 0.79 \\
\hline \multirow{4}{*}{16} & Min & 0.85 & 0.75 & 1.17 & 1.06 \\
\hline & Avg & 0.98 & 1.00 & 1.97 & 2.08 \\
\hline & Max & 1.16 & 1.31 & 3.14 & 3.69 \\
\hline & StD & 0.09 & 0.17 & 0.65 & 0.83 \\
\hline \multirow{4}{*}{24} & Min & 0.91 & 0.93 & 1.16 & 1.09 \\
\hline & Avg & 1.15 & 1.16 & 2.06 & 2.19 \\
\hline & $\operatorname{Max}$ & 1.72 & 1.60 & 3.49 & 4.08 \\
\hline & StD & 0.20 & 0.21 & 0.70 & 0.91 \\
\hline \multirow{4}{*}{32} & Min & 1.05 & 1.04 & 1.33 & 1.26 \\
\hline & Avg & 1.51 & 1.49 & 2.24 & 2.41 \\
\hline & $\operatorname{Max}$ & 2.52 & 2.63 & 3.71 & 4.51 \\
\hline & StD & 0.45 & 0.45 & 0.74 & 1.02 \\
\hline
\end{tabular}

does not seem to have a big impact in the performance, however it still leaves room for further improvements, since the difference between local and batched scheduling is higher in the FS design than in the NS design.

\section{Conclusions and Further Work}

Local and batched scheduling are arguably two of the most well-known tabling scheduling strategies. The major difference between both is that local scheduling propagates answers only after all answers are found, while batched scheduling propagates answers immediately after they are found. Batched scheduling is a useful strategy in tabled logic programs that require an eager propagation of answers and/or do not require the complete set of answers to be found. In this work, we have presented the PAC strategy, which is a simple and novel approach for combining the FS design with batched scheduling. PAC splits answer representation from answer propagation, and allows the first to be publicly shared among threads while the second to be private to each thread.

Experimental results in worst-case scenarios showed that, on average, the PAC strategy does not seem to have a big impact in the performance, however 
it still leaves room for further improvements specially in the extra structures required to control the propagated answers. Further work will include the usage of time-stamped tries to minimize the search for the propagated answers and new real-world problems that will allow us to improve and consolidate our framework.

\section{Acknowledgments}

This work is partially funded by the North Portugal Regional Operational Programme (ON.2 - O Novo Norte) and by the National Strategic Reference Framework (NSRF), through the European Regional Development Fund (ERDF) and

the Portuguese Foundation for Science and Technology (FCT), within projects NORTE-07-0124-FEDER-000059 and UID/EEA/50014/2013.

\section{References}

1. Areias, M., Rocha, R.: An Efficient and Scalable Memory Allocator for Multithreaded Tabled Evaluation of Logic Programs. In: International Conference on Parallel and Distributed Systems. pp. 636-643. IEEE Computer Society (2012)

2. Areias, M., Rocha, R.: Towards Multi-Threaded Local Tabling Using a Common Table Space. Journal of Theory and Practice of Logic Programming 12(4 \& 5), 427-443 (2012)

3. Areias, M., Rocha, R.: A Simple and Efficient Lock-Free Hash Trie Design for Concurrent Tabling. In: Technical Communications of the International Conference on Logic Programming (2014)

4. Areias, M., Rocha, R.: A lock-free hash trie design for concurrent tabled logic programs. International Journal of Parallel Programming pp. 1-21 (2015)

5. Chen, W., Warren, D.S.: Tabled Evaluation with Delaying for General Logic Programs. Journal of the ACM 43(1), 20-74 (1996)

6. Freire, J., Swift, T., Warren, D.S.: Beyond Depth-First: Improving Tabled Logic Programs through Alternative Scheduling Strategies. In: International Symposium on Programming Language Implementation and Logic Programming. pp. 243-258. No. 1140 in LNCS, Springer (1996)

7. Liang, S., P.Fodor, Wan, H., M.Kifer: OpenRuleBench: An Analysis of the Performance of Rule Engines. In: Internacional World Wide Web Conference. pp. 601-610. ACM (2009)

8. Marques, R., Swift, T.: Concurrent and Local Evaluation of Normal Programs. In: International Conference on Logic Programming. pp. 206-222. No. 5366 in LNCS, Springer (2008)

9. Ramakrishnan, I.V., Rao, P., Sagonas, K., Swift, T., Warren, D.S.: Efficient Access Mechanisms for Tabled Logic Programs. Journal of Logic Programming 38(1), 3154 (1999)

10. Sagonas, K., Swift, T.: An Abstract Machine for Tabled Execution of Fixed-Order Stratified Logic Programs. ACM Transactions on Programming Languages and Systems 20(3), 586-634 (1998)

11. Wielemaker, J.: Native Preemptive Threads in SWI-Prolog. In: International Conference on Logic Programming. pp. 331-345. No. 2916 in LNCS, Springer (2003) 